\title{
Upgraded Cameras for the H.E.S.S. Imaging Atmospheric Cherenkov Telescopes
}

Gianluca Giavitto ${ }^{\mathrm{a}}$, Terry Ashton ${ }^{\mathrm{b}}$, Arnim Balzerc ${ }^{\mathrm{c}}$, David Berge ${ }^{\mathrm{c}}$, Francois Brun ${ }^{\mathrm{d}}$, Thomas Chaminade $^{\mathrm{d}}$, Eric Delagnes ${ }^{\mathrm{d}}$, Gerard Fontaine ${ }^{\mathrm{f}}$, Matthias Füßling ${ }^{\mathrm{a}}$, Berrie Giebels ${ }^{\mathrm{f}}$, Jean-Francois Glicenstein ${ }^{\mathrm{d}}$, Tobias Gräber ${ }^{\mathrm{a}}$, Jim Hinton ${ }^{\mathrm{g}}$, Albert Jahnke ${ }^{\mathrm{g}}$, Stefan Klepser ${ }^{\mathrm{a}}$, Marko Kossatz ${ }^{\mathrm{a}}$, Axel Kretzschmann ${ }^{\mathrm{a}}$, Valentin Lefranc ${ }^{\mathrm{a}, \mathrm{e}}$, Holger Leich ${ }^{\mathrm{a}}$, Hartmut Lüdecke ${ }^{\mathrm{a}}$, Iryna Lypova ${ }^{\mathrm{a}}$, Pascal Manigot ${ }^{\mathrm{f}}$, Vincent Marandon ${ }^{\mathrm{g}}$, Emmanuel Moulin ${ }^{\mathrm{d}}$, Mathieu de Naurois ${ }^{\mathrm{f}}$, Patrick Nayman ${ }^{\mathrm{e}}$, Marek Penno ${ }^{\mathrm{a}}$, Duncan Ross ${ }^{\mathrm{b}}$, David Salek ${ }^{\mathrm{c}}$, Markus Schade ${ }^{\mathrm{a}}$,

Thomas Schwabㅁ, Rachel Simonic, Christian Stegmann ${ }^{\mathrm{a}}$, Constantin Steppa ${ }^{\mathrm{a}}$, Julian

Thornhill $^{\mathrm{b}}$, and Francois Toussnel ${ }^{\mathrm{e}}$

${ }^{a}$ DESY, D-15738 Zeuthen, Germany

${ }^{\mathrm{b}}$ Department of Physics and Astronomy, The University of Leicester, University Road, Leicester, LE1 7RH, United Kingdom

${ }^{\mathrm{c}}$ GRAPPA, Anton Pannekoek Institute for Astronomy, University of Amsterdam, Science Park 904, 1098 XH Amsterdam, The Netherlands

${ }^{\mathrm{d} D S M} / \mathrm{Irfu}$, CEA Saclay, F-91191 Gif-Sur-Yvette Cedex, France

eLPNHE, Université Pierre et Marie Curie Paris 6, Université Denis Diderot Paris 7, CNRS/IN2P3, 4 Place Jussieu, F-75252, Paris Cedex 5, France

${ }_{\mathrm{f}}^{\mathrm{L}}$ aboratoire Leprince-Ringuet, Ecole Polytechnique, CNRS/IN2P3, F-91128 Palaiseau, France gMax-Planck-Institut für Kernphysik, P.O. Box 103980, D 69029 Heidelberg, Germany

\begin{abstract}
The High Energy Stereoscopic System (H.E.S.S.) is an array of five imaging atmospheric Cherenkov telescopes, sensitive to cosmic gamma rays of energies between $\sim 30 \mathrm{GeV}$ and several tens of TeV. Four of them started operations in 2003 and their photomultiplier tube (PMT) cameras are currently undergoing a major upgrade, with the goals of improving the overall performance of the array and reducing the failure rate of the ageing systems. With the exception of the 960 PMTs, all components inside the camera have been replaced: these include the readout and trigger electronics, the power, ventilation and pneumatic systems and the control and data acquisition software. New designs and technical solutions have been introduced: the readout makes use of the NECTAr analog memory chip, which samples and stores the PMT signals and was developed for the Cherenkov Telescope Array (CTA). The control of all hardware subsystems is carried out by an FPGA coupled to an embedded ARM computer, a modular design which has proven to be very fast and reliable. The new camera software is based on modern $\mathrm{C}++$ libraries such as Apache Thrift, ØMQ and Protocol buffers, offering very good performance, robustness, flexibility and ease of development. The first camera was upgraded in 2015, the other three cameras are foreseen to follow in fall 2016. We describe the design, the performance, the results of the tests and the lessons learned from the first upgraded H.E.S.S. camera.
\end{abstract}

Keywords: HESS upgrade, PMT signal readout, Cherenkov telescope, VHE gamma-ray astronomy, NECTAr

Further author information: (Send correspondence to G. G.)

G. Giavitto: E-mail: gianluca.giavitto@desy.de, Telephone: +49 (0)33762 7-7457

B.B.A.: E-mail: bba@cmp.com, Telephone: +33 (0)198765432

Ground-based and Airborne Instrumentation for Astronomy VI, edited by Christopher J. Evans, Luc Simard, Hideki Takami Proc. of SPIE Vol. 9908, 99082H · (c) 2016 SPIE · CCC code: 0277-786X/16/\$18 · doi: 10.1117/12.2231865 

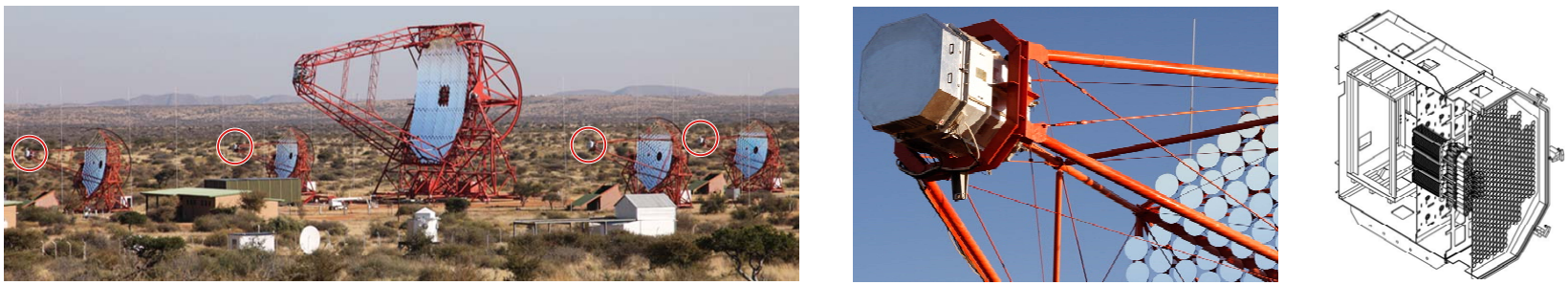

Figure 1. Left: bird's eye view of the H.E.S.S. array. The four smaller CT1-4 telescopes surround the bigger CT5. In circles, the cameras to be upgraded. The bigger light brown building with the green roof is the control building, the smaller light brown buildings close to the telescopes are the camera shelters. Each telescope is equipped with a white container where several electrical cabinets controlling the motors are placed. Center: picture of the first "flight" of the H.E.S.S. I Upgrade camera. Right: half section of the mechanical structure of the camera, showing four drawers.

\section{INTRODUCTION}

The first Cherenkov telescopes (CT) of the H.E.S.S. array were the $12 \mathrm{~m}$ diameter CT1-4, which were built and commissioned between 2002 and 2004 at the H.E.S.S. site in the Khomas highlands in Namibia. CT1-4 are also known as the "H.E.S.S. I array". A fifth, 28-m diameter telescope was built in 2012 in the middle of the square H.E.S.S. I array (see Figure 1). This new telescope, called CT5, was built with the goal of lowering the minimum gamma-ray energy threshold of H.E.S.S. from $\sim 100 \mathrm{GeV}$ down to $\sim 30 \mathrm{GeV}$. To reach that goal, CT5 has the largest mirror surface area of all operational and planned Cherenkov telescopes: this allows it to trigger on smaller air showers, and as a result it operates at a about ten times the event rate than the older, smaller CT1-4. Its camera features more modern readout electronics ${ }^{1,2}$ than the older CT1-4 cameras, in order to cope with the higher trigger rate.

One of the main reasons to upgrade the 14-year-old CT1-4 cameras ${ }^{3}$ is precisely that of reducing the dead time of the array when is operated together with CT5. The readout time of the original CT1-4 cameras is $\sim 450 \mu \mathrm{s}$ which leads to negligible losses in case of typical H.E.S.S. I array trigger rates $(200-300 \mathrm{~Hz})$, but when CT1-4 trigger in coincidence with CT5, array trigger rates of $1.5 \mathrm{kHz}$ or more can occur, depending on the trigger threshold setting. This can lead to a substantial fraction of events losing their stereoscopic quality because CT1-4 are in readout state, thus unavailable for triggering.

The most important element of the upgraded cameras contributing to the reduction of the dead time is the readout, built around the NECTAr analog memory chip. ${ }^{4}$

An equally important reason to upgrade the old CT1-4 cameras is to stop the inevitable increase in failures due to the ageing of the electronics, connectors and other critical parts that have been exposed for 14 years to the harsh condition of the Namibian site.

To achieve a reduction in the failure rate, besides renewing the electronics, new cabling, ventilation, power supply and pneumatics designs were adopted. The first upgraded camera has been deployed in 2015, and is currently taking data.

Additionally, this upgrade offers the opportunity to improve the performance of the array. For instance, since the new readout can sustain much higher trigger rates, it is possible to reduce the trigger thresholds of CT1-4, thereby extending their sensitivity to gamma-ray showers of energies below $100 \mathrm{GeV}$. Other characteristics of the upgraded trigger and readout could also improve the performance of CT1-4 at higher energies.

\section{CAMERA COMPONENTS}

The camera upgrade replaced almost every component of the camera, except the photomultiplier tubes (PMTs), their high voltage bases and the calibration units. A scheme of the camera logical blocks, including electronic and mechanical parts, their architecture and interplay, can be seen in Figure 2.

Most of the new devices designed for this upgrade are all equipped with an FPGA (Cyclone IV) and an ARMv5-based $\mu$ Computer Module (Stamp9G45). The latter controls and communicates with the former by 


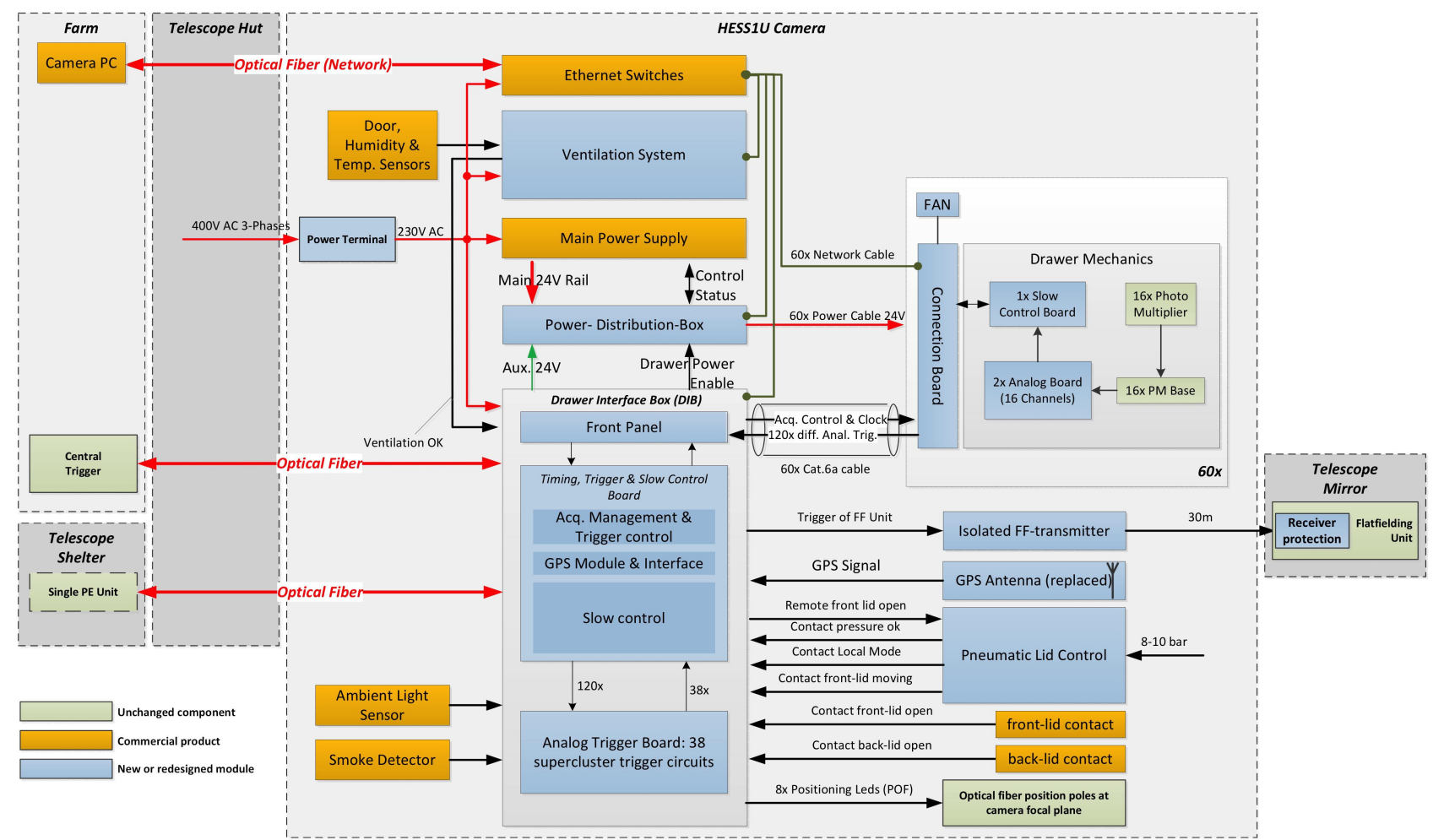

Figure 2. Schematic diagram illustrating the logical blocks of the H.E.S.S. I Upgrade. The few original H.E.S.S. I systems are denoted by green boxes. Commercial, off-the-shelf components are marked in orange. The dashed boxes surrounding components groups correspond to actual physical locations: the bigger "HESS1U Camera" box corresponds to the camera itself, the "telescope shelter" is the shelter in which the camera is parked during the day, the "telescope hut" is the white container mounted on the structure of the telescope, the "farm" is the server room located inside the control building.

means of a fast 100MBit/s memory bus, with a word width of $16 \mathrm{bit}$. This solutions allows for maximum flexibility and easy control and communication with all subsystems, improving the overall resilence of the camera.

\subsection{Front-end electronics}

The camera front-end electronics is where Cherenkov light from particle showers in the atmosphere is detected and digitized. The light detection is carried out by 960 PMTs, operated at a gain of $2 \times 10^{5}$. The modules responsible for the amplification and readout of the PMT signals are called drawers. There are 60 drawers in a camera, arranged in a $9 \times 8$ rectangular matrix, with each corner of the matrix devoid of 3 drawers. A drawer consists of two analog boards, each hosting 8 analog channels, connected to a slow control board, which is in turn connected to a connection board. The 60 drawers are supported by a mechanical structure called the sandwitch plate, and the connection boards screwed onto the back side of that plate. Each connection board is the end point of the data, trigger and power networks originating from the back-end.

\subsubsection{The analog boards}

When a PMT detects Cherenkov photons from an atmospheric shower, it generates an electrical pulse of current, which is fed into the first upgraded component of the camera, the analog board. The signal from the PMT is typically a few nanoseconds long and a has an amplitude varying from $1 \mathrm{mV}$ to more a few $\mathrm{V}$, depending on the number of photons detected. It is sent to the analog board via a $15 \mathrm{~cm}$ long coaxial cable, and is there terminated with $R=50 \Omega$. The single-ended, AC coupled signal is then pre-amplified, split into three branches and further amplified by low noise single-ended to differential amplifiers. Two of these three signal branches are routed to the inputs of the NECTAr chip, for sampling and digitalization. Their total amplification factors are 15.1 (high gain, HG) and 0.68 (low gain, LG). The NECTAr chip inputs have a range of 2V. An adjustable 
constant common-mode offset is added to the electrical signals just before it enters the chip, in order to account for a possible undershoot and drifts in the baseline due to temperature effects. Its default value is about $205 \mathrm{mV}$, but it is automatically calibrated at the beginning of observation.

The third signal branch is amplified by a factor 45 and routed to a comparator, the output of which goes directly into the FPGA, where is sampled at $800 \mathrm{MHz}$. This signal, referred to as the level 0 (L0) trigger signal, is further manipulated and sent to the back-end electronics, which generates the camera trigger, or level 1 (L1) trigger.

\subsubsection{The readout}

The readout is built around the NECTAr chip, a fast sampler and digitizer chip. It integrates three functions:

- An analogue memory based on a switched capacitor array in which the analogue signal is sampled and stored at very high rate $(1 \mathrm{GS} / \mathrm{s})$;

- A 12-bit $21 \mathrm{MS} / \mathrm{s}$ ADC digitizing the data stored in the analogue memory;

- A serializer, which sends the digital data downstream to the FPGA.

Each chip hosts two switched capacitor arrays with a depth of 1024 cells each. Operations are simultaneous on the 2 channels. In normal operations only a small fraction of the cells inside a region of interest (usually 16 cells) are read out. The sampling is stopped by an external trigger signal, which must be synchronous to the analogue signal one whishes to digitize. When reading 16 cells, the nominal dead time of a NECTAr chip is lower than $\sim 2 \mu \mathrm{s}$. However, The overall dead time of the upgraded H.E.S.S. camera readout is $\sim 5.5 \mu \mathrm{s}$, because the way the chip is read out by the FPGA requires a minimum safe interval between two events greater than the nominal dead time.

The typical bandwidth of the NECTAr chip analog inputs as reported in its datasheet ${ }^{5}$ is between 300 and $500 \mathrm{MHz}$. Care was taken to use analog components matching that value. The overall end-to-end bandwidth of the readout was measured by a pure sine wave frequency scan to be $\sim 330 \mathrm{MHz}$. One can appreciate the effect of such a high bandwidth in the readout pulse shape shown in figure 3, where the FWHM is less than 3 ns.

The analog part of the readout was developed to have very low noise. The pedestal noise, measured as the RMS of the value of a single cell is around 4 ADC counts, or $2 \mathrm{mV}$. At the nominal PMT gain of $2 \times 10^{5}$, this corresponds to 0.32 photoelectrons.

The linearity of the high gain and low gain channels are on average better than $2 \%$. The cross talk between two channels within one NECTAr chip and between channels on different nectar chips is typically less than $1.5 \%$, for high gain and $6 \%$ for low gain, see Figure 4 .

The high gain linear range is between 0 and 200 photoelectrons, and the low gain one is between 30 and 4200 photoelectrons; the overall dynamic range of this readout is therefore greater than $80 \mathrm{~dB}$. The high to low gain ratio remains constant at around 22 between 30 and 200 photoelectrons, see Figure 4.

\subsection{The slow control board}

The slow control board is where the FPGA and the ARM micro computer are located. The FPGA reads out the digitized signals from the NECTAr chips and transmits it to ARM cpu, which buffers it and sends it to a central camera computer via TCP/IP using ØMQ sockets. ${ }^{6}$ The data (both readout and slow control) is transmitted via ethernet by means of a standard Cat. 6 cable. The trigger L0 signals, fast readout control and clock distribution are carried on standard Cat. $6_{A}$ ethernet cables. The power network delivers $24 \mathrm{~V}$ DC to each drawer on industry standard 4-wire cables, ending with M8 connectors. The connection board has therefore 2 ethernet and one 4-pin M8 plug. The main step-down DC-DC converter is also hosted on the connection board, thus removing one major source of noise and heat from the inner part of the drawer.

\subsection{Back-end electronics}

The back-end electronics is deployed inside one $19 \mathrm{in}$. rack located in the posterior van of the camera (see Figure 5 and 6 ). New components developed specifically for this upgrade are described in the following. 

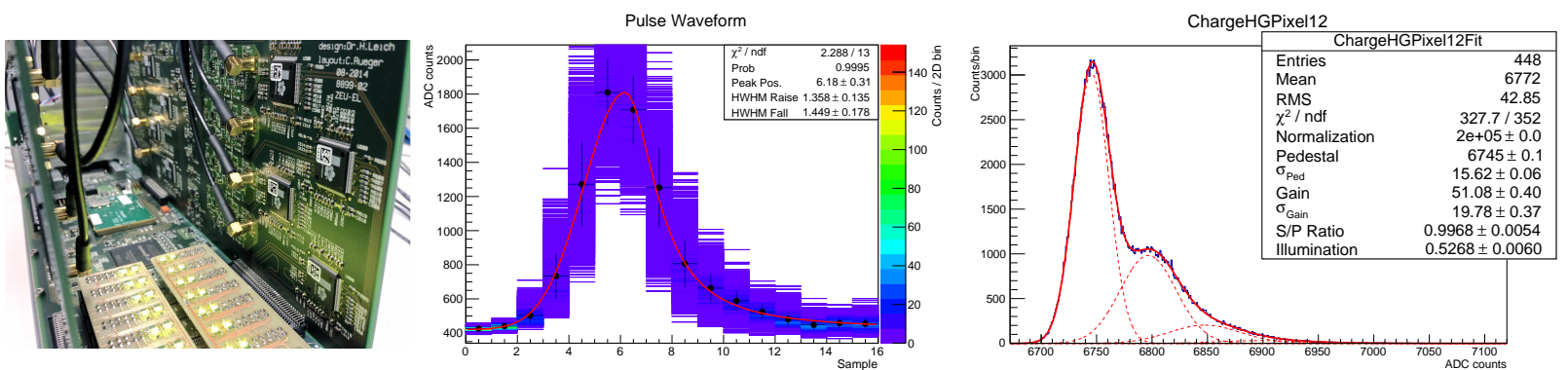

Figure 3. Left: View into a new drawer, with analog front-end boards at the right and left, and drawer slow control board at the bottom. The analog boards contain the NECTAr chips (with the flower logo), the slow control board has test loads mounted at the PMT bases connectors (green lights). Behind them, the big black chip is the FPGA, and the green add-on board at the back is the ARM computer module. Center: typical PMT pulse from a calibration source, as it is recorded by the readout. Right: Results of a PMT gain calibration. The first peak is the pedestal peak, the following ones are the one-, two-, ... photoelectrons peaks. The electronic intrinsic noise is the width of the pedestal peak, and it is about 15.6 ADC counts or about one third of a photoelectron.
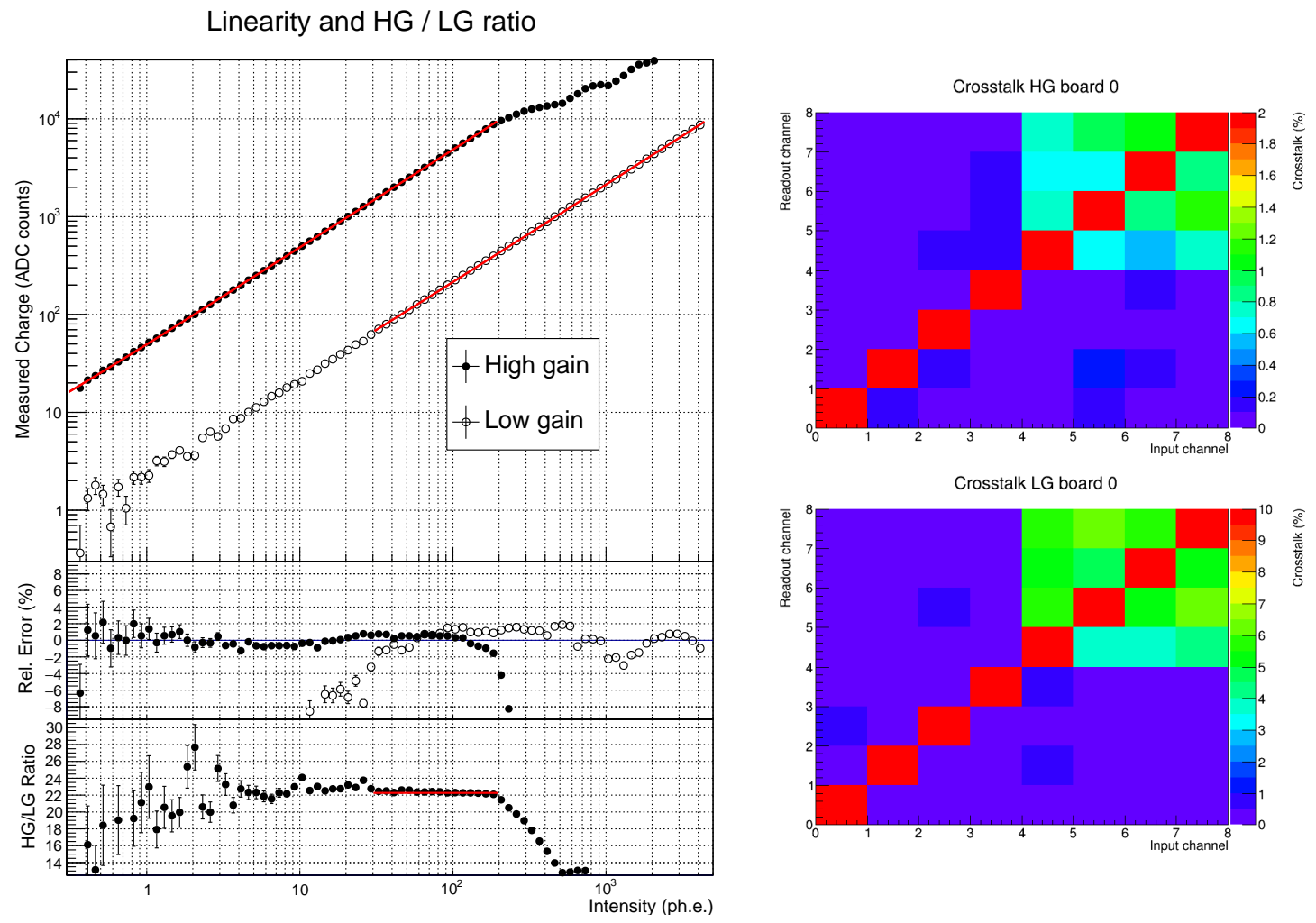

Figure 4. Left: Summary of the linearity results for a typical channel. In the top frame, response curves are shown of both high gain (black filled circles) and low gain (empty circles) readout channels. Right: Relative cross talk inside one analog board at the typical pulse amplitude (about 20 ph.e. for HG, 200 ph.e. for LG). The higher cross-talk in the channels $4-7$ is due to the routing layout of the analog board, and not to the NECTAr chips. 


\subsubsection{Drawer interface box}

The most important and complex component of the back-end electronics is a 3-unit module called drawer interface box (DIB). From a functional point of view, the DIB is the central hub of the whole camera and is responsible for the following function:

- Trigger interface to 60 drawers and to the array central trigger

- Clock distribution to the drawers

- Camera (L1) trigger generation

- Interface to the following camera components

Flatfielding calibration unit

Single photoelectron calibration unit

Camera position LEDs on the lid

Ambient light sensor

Smoke detector

Ventilation system

- Protection of PMT and camera electronics

High Voltage interlock

Drawer power enable

- GPS timestamping of events

The DIB contains 3 interconnected boards: a front panel, a main board and an analog trigger board. The front connection board hosts the 60 sockets delivering a $10 \mathrm{MHz}$ clock signal, the acquisition control and receiving the L0 trigger signals from the 60 drawers. The clock and acquisition control are transmitted using length-coded LVDS signals. The two L0 trigger (one for each analog board) from the drawers are height-coded analog signals. These signals are carried on the four pairs of a standard Cat. $6_{A}$ ethernet cable. The main board hosts a separated analog trigger board to which the L0 trigger signals are sent. This additional board is responsible of the camera (L1) local trigger, but it is the main board that communicates with the central trigger of the array, by means of a fiber interface.

Many sensors were deployed inside and outside the camera for ambient light, temperature, smoke and humidity. Their signals are fed to a new safety interlock system to guarantee safe camera operations for both shift crew and hardware. This security interlock logic is implemented at the level of the FPGA firmware: in case of errors or failures it turns off the power of the drawers and closes the camera lid, without the need of any software command.

\subsubsection{Power distribution box}

The power is generated by the main power supply, which is a commercial 3-module unit from TDK Lambda which delivers the $24 \mathrm{~V}$ and $\sim 80 \mathrm{~A}$ needed by the camera. Three modules share the load, but only two are actually needed, one serves as a hot spare. The power is distributed to the 60 drawers by the Power Distribution $B o x$ (PDB), a 64 channel power switch which monitors constantly the consumption of current of each drawer, and is able to shut the power off if it detects an overcurrent. 

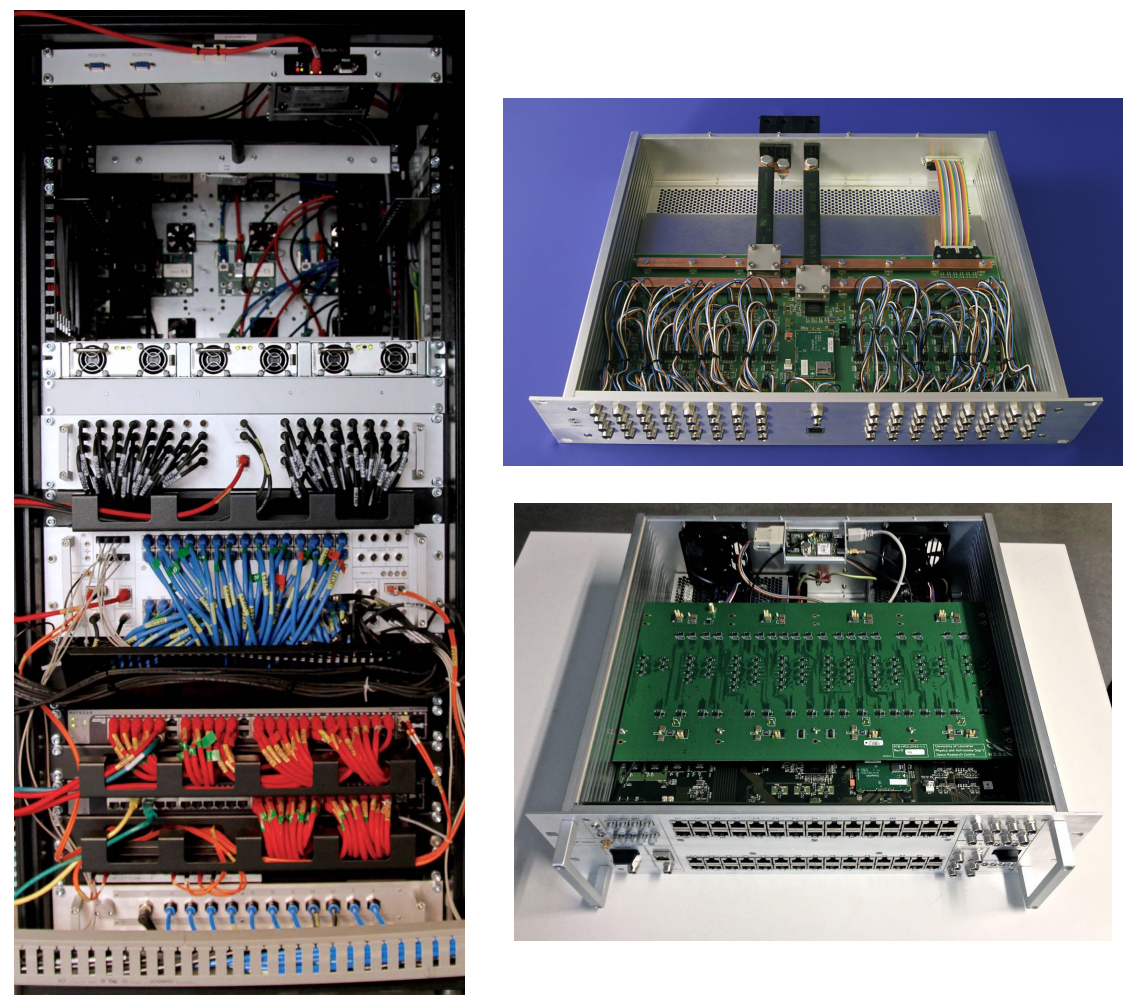

Figure 5. Left: Picture of the back-end rack. From top to bottom: ethernet-controlled main power switch (1 unit), empty space (6 units), then commercial 1-unit TDK Lambda $24 \mathrm{~V}$ DC power supply, power distribution box (2 units), drawer interface box (3 units) 2 48-ports NETGEAR 10GB/s network switches, pneumatic control box. The red ethernet cables are for the data network, the blue ethernet cables for the trigger, the black cables for the power. Right: Picture of the power distribution box (top) and the drawer interface box (bottom), with their lids removed to show the boards inside.

\subsection{Camera trigger scheme}

The camera trigger is kept very similar to what it was in the old camera, namely that a signal coincidence between $N$ pixels is required in any of 38 overlapping "trigger sectors" for the camera to trigger.

The path of the signal along the trigger branch is the following: the PMT pulse is amplified and fed to a comparator, to produce the L0 trigger. The binary L0 signal from every pixel on the drawer is sampled at $800 \mathrm{MHz}$ by the FPGA. The number of pixels with L0 active in each of the two analog boards on the drawer are sent as height-coded analog signal to the drawer interface box, over two wire pairs of a Cat. $6 A_{A}$ ethernet cable. The steps of the height coding have an amplitude of $33 \mathrm{mV}$. In the drawer interface box these signals are isochronously routed to the 38 overlapping trigger sectors analog summators, located on the analog trigger board. These summators build an analog sum of all L0 signals inside a sector. As mentioned before, the 60 drawers are arranged to occupy the central positions of a $9 \times 8$ bidimensional array, and the sectors overlap in width by one half-drawer and in height by one full drawer, so the signal from each half drawer is routed to at most 4 sectors. The output of the each sector sum goes to a comparator, where a common threshold signal (corresponding to $N$ pixels fired) is applied. The 38 comparator outputs are finally combined in an OR logic to give the camera trigger.

The only difference between this design and the original one is the fact that the L0 trigger comparator output is sampled, albeit at a very high frequency of $800 \mathrm{MHz}$, whereas in the old design it was not. This allows for greater flexibility and even for more complex trigger scenarios to be implemented, so it could work as a testbed for concepts put forward for the next generation of Cherenkov telescopes (e.g. digital trigger ${ }^{7}$ ). With the present scheme, it is expected that the inefficiency inherent to sampling these pulses can be mitigated by lowering the pixel thresholds, while the pixel jitter introduced is negligible (1.25 ns). Indeed, several threshold scan with the first upgraded camera have shown that the performance of this new trigger is comparable to that of the old one. 


\subsection{Ventilation}

The ventilation concept has been redesigned from the ground up: a new ventilation and heating system was constructed on the new back door of the camera, with a single fan, replacing the many fans previously mounted on the camera sides. Pneumatic cylinders were added to be able to open and close the heavier back door, and the control unit of the entire camera ventilation was renewed as well with a new PLC module.

The new ventilation system is designed to steadily inject filtered and (optionally) heated air into the camera. It causes a steady overpressure in the entire camera body, and leads to a constant air flow through the drawers and all other small openings of the camera. This way a homogeneous cooling is achieved, and dust is prevented from entering the camera. The option to heat the incoming air is used to lower the humidity after periods of shutdown.

The volume of air exchanged by the ventilation is $>1300 \mathrm{~m}^{3} / \mathrm{hr}(>360 \mathrm{~L} / \mathrm{s})$. The data acquired so far shows that such air flow can keep the drawers at a temperature of $\sim 32^{\circ} \mathrm{C}$, with a gradient of $\pm 5{ }^{\circ} \mathrm{C}$ along the top-bottom direction. Both absolute temperature and temperature gradient have no measurable effect on the data and on the trigger efficiency.

\subsection{Camera network and control software}

Drawers, drawer interface box, power distribution box and ventilation system are all connected to the main camera server via Ethernet connections. The camera network carries data to/from all the aforementioned devices. All slow-control commands are called remotely from the central camera server and are independent from one another, forming a star-like network topology. Single devices all have $100 \mathrm{Mbit} / \mathrm{s}$ copper-based connection to a central switch, which is then connected to the main camera server by means of a $10 \mathrm{Gbit} / \mathrm{s}$ optical fiber connection. As a novelty, the camera server is not located inside the camera, but remotely in the control building (see Fig. 2), easing its maintenance.

The presence of an ARM-based computer module running Linux on all of the camera systems (with the exception of the ventilation system), makes communication between single components very easy. In order to reduce the slow control and data acquisition software development time, several open source software solutions were adopted.

The operating system running on the Stamp9G45 is a version of Linux, build using the Yocto embedded Linux build system, ${ }^{8}$ with the kernel module provided by the manufacturer.

Slow control communication is implemented using the Apache Thrift, a lightweight and performant RPC framework. ${ }^{9}$ Data transfer was implemented using the $\varnothing \mathrm{MQ}^{6}$ smart socket message-passing library as a transport backbone. Raw event data messages are serialized via an optimized custom protocol, and monitoring data messages are serialized using the Google Protocol Buffers library. ${ }^{10}$

These technologies allowed for a robust implementation of network-based slow control and event acquisition to be developed by a very small team of two people in short timescales. Evaluation tests showed that the Thrift RPC framework can sustain rates of 10000 requests per second for tens of hours without a single failure, in a busy, complex network. Bulk data transfer tests using the ØMQ library have saturated the current $1 \mathrm{~GB} / \mathrm{s}$ data connection, and have exceeded $5 \mathrm{~GB} / \mathrm{s}$ on a $10 \mathrm{~GB} / \mathrm{s}$ connection. In real-life conditions, the cameras never need to sustain the event rates needed for link saturation.

\subsection{Tests}

Due to the scale and scope of the upgrade, testing the new electronics during its prototyping and its mass production stages is very important. Four main test setups were built: a test bench for the drawer production tests, a black box with a light source to test the drawers with the PMTs attached, a minicamera with four drawers, and a full copy of the camera.

The upgrade required the production and testing of about 270 drawers. The drawers are the most complex item of the upgrade, therefore an automatic test bench was specifically set up to ease their testing. An 8-channel pulse generator was designed and build for this purpose. Each individual channel on one such pulse generator can deliver a negative pulse with rise and fall times $\sim 1 \mathrm{~ns}$, with programmable attenuation up to $-54 \mathrm{~dB}$ with $1 \mathrm{~dB}$ 

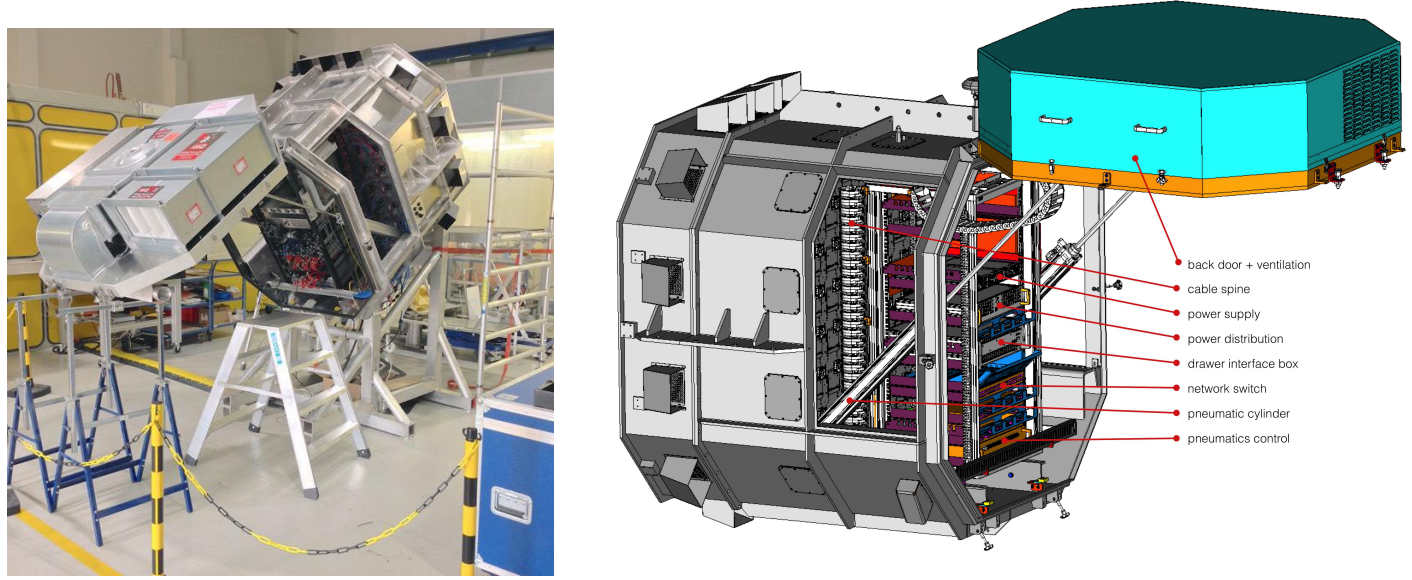

Figure 6. Reproduction of a CT1-4 camera used for test purposes at DESY in Zeuthen. Left: Photo with uncovered back door, such that ventilation components (filters, fans, heating) can be seen. Inside the camera, the rack is mounted with various units installed and blue (trigger) and red (data) cables connecting them. The camera is mounted with the same inclination as in Namibia, to allow for realistic training of deployment. Through the side opening of the camera body, drawer connection boards with their fans can be seen. Right: Labeled sketch of the camera, visualising the layout of the rack units

steps (from $\sim 300 \mathrm{mV}$ down to $\sim 0.6 \mathrm{mV}$ ), programmable delay (up to $64 \mathrm{~ns}$ in $0.25 \mathrm{~ns}$ steps) and programmable width (from $2 \mathrm{~ns}$ up to $62 \mathrm{~ns}$ in $0.25 \mathrm{~ns}$ steps). Furthermore the generator is equipped with a trigger input, a trigger output and a gate, plus a RJ45 connector to mimick the trigger signal protocol of the upgraded camera. Two of these devices equip the test bench, and permitted testing the 16 channels of a drawer simultaneously. More than 300 single unit tests were performed per test run. The tests included checks of basic functionality, pedestal noise, linearity, cross-talk, and trigger path tests. Two test benches were set up at DESY in Zeuthen for the mass production tests of the drawers.

In any case, several of the tests not requiring an external input can be repeated anytime by the ARM module on the drawer itself. Therefore, it is possible to test most of the features of a drawer even after its installation on the camera.

One further test bench, the "black box", was used extensively during prototyping; it is now located at the H.E.S.S. site, where it was used during the deployment of CT1 and is occasionally for inspecting malfunctioning drawers from remote. It consists of a dark box hosting a drawer and a light source. One can therefore test the PMTs in dark conditions also during the day.

A bigger dark box with a 4-drawer "mini camera", including PMTs, was constructed with the goal of having a minimal test camera to create a realistic dark trigger setup. A copy of the H.E.S.S. data acquisition system $\left(\mathrm{DAQ}^{11}\right)$ was also attached to this mini camera and the original state transitions of the system were emulated.

In order to test the interplay of the new components as good as possible before deployment, a replica of a H.E.S.S. I camera was produced and assembled in Zeuthen (Fig. 6). Even without the photomultiplier tubes (PMTs) available, the trigger chain, camera-internal network, cable mapping, ventilation, slow control, power supply and mechanical integration could thus be tested to a good extent.

Furthermore, the whole trigger path was tested, from the L0 trigger pixel comparator right after the PMT up to the sector comparator and the camera L1 trigger logic. At each position in the trigger chain dedicated scope measurements and special rate scans were performed to assess the level of noise and the purity and inefficiency of the trigger. The measurements showed that the $N$-majority logic implementation has negligible noise and that for every setting of $N$ exists a threshold setting at which all $N$-multiplicity trigger are issued and no $N-1$-multiplicity signal can trigger. 

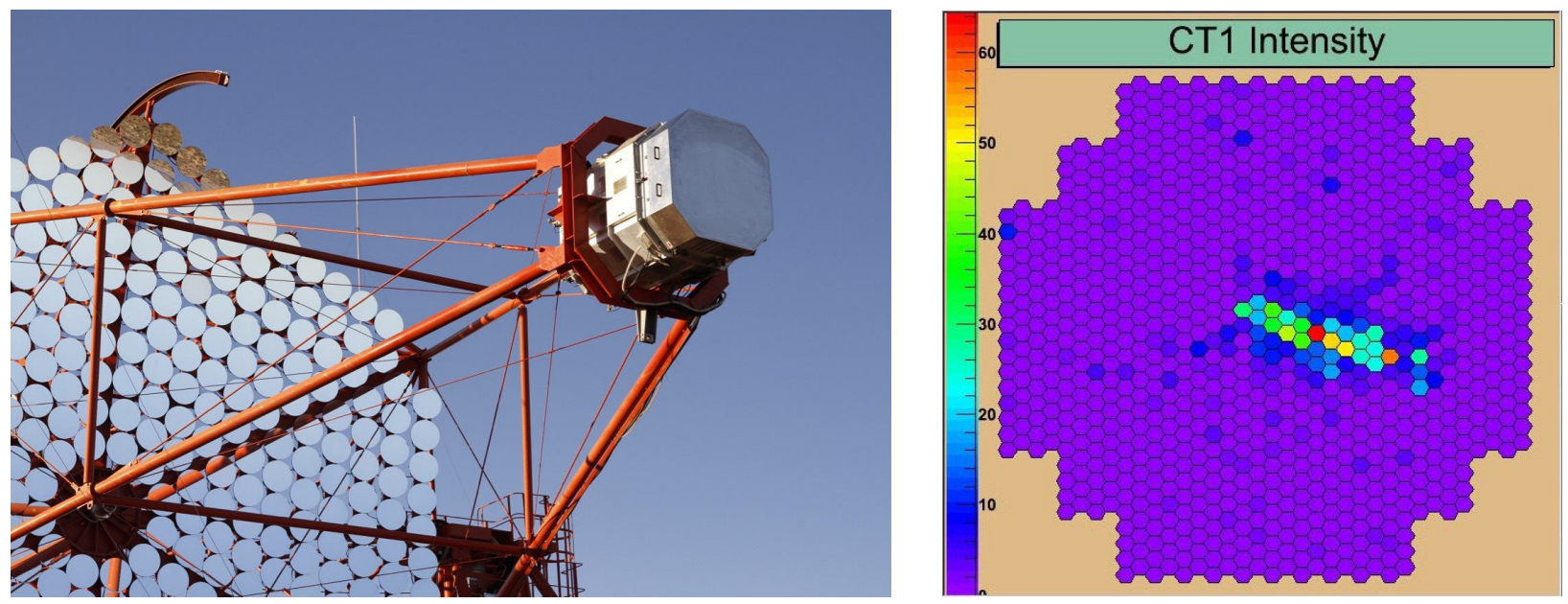

Figure 7. Left: The upgraded H.E.S.S. CT1 camera after installation in July 2016. Right: One of the first Cherenkov showers recorded with the upgraded camera.

\section{STATUS OF CT1 AND OUTLOOK}

The first camera (CT1) was upgraded in summer 2015 (Fig. 7, left). The plain hardware deployment could be finished after only 3 weeks; the on-site software commissioning led to the first gamma-ray shower images in August 2016 (Fig. 7, right). After precise calibration and flat-fielding campaigns of gains, readout delays, trigger thresholds for all pixels, and a moderate level of remote post-deployment commissioning campaign to adjust the camera to the subtleties of reliable array operation, the camera is participating reliably in regular nightly data taking since March 2016.

The remaining three cameras have been produced in the meanwhile and are foreseen to be deployed in one campaign in fall 2016. Full array observations might commence in spring 2017.

Besides the improved dead time, the design of the H.E.S.S. I Upgrade opens many possibilities to optimise the trigger logic, front-end readout window and time information of pulses. The upgraded H.E.S.S. cameras can therefore be expected to improve the sensitivity of H.E.S.S., but also continue to provide a test bed for various technical innovations that are being discussed in the framework of CTA R\&D efforts.

\section{ACKNOWLEDGMENTS}

The support of the Namibian authorities and of the University of Namibia in facilitating the construction and operation of H.E.S.S. is gratefully acknowledged, as is the support by the German Ministry for Education and Research (BMBF), the Max Planck Society, the German Research Foundation (DFG), the French Ministry for Research, the CNRS-IN2P3 and the Astroparticle Interdisciplinary Programme of the CNRS, the U.K. Science and Technology Facilities Council (STFC), the IPNP of the Charles University, the Czech Science Foundation, the Polish Ministry of Science and Higher Education, the South African Department of Science and Technology and National Research Foundation, and by the University of Namibia. We appreciate the excellent work of the technical support staff in Berlin, Durham, Hamburg, Heidelberg, Palaiseau, Paris, Saclay, and in Namibia in the construction and operation of the equipment.

\section{REFERENCES}

[1] Bolmont, J. et al., "The camera of the fifth H.E.S.S. telescope. Part I: System description," NIM A 761, 46-57 (Oct. 2014).

[2] Delagnes, E., Degerli, Y., Goret, P., Nayman, P., Toussenel, F., and Vincent, P., "SAM: A new GHz sampling ASIC for the H.E.S.S.-II front-end electronics," NIM A 567, 21-26 (Nov. 2006).

[3] Vincent, P. et al., "Performance of the H.E.S.S. cameras," Proc. 28th ICRC (2003). 
[4] Naumann, C. L. et al., "New electronics for the Cherenkov Telescope Array (NECTAr)," NIM A 695, 44 (2012).

[5] Delagnes, E., "Specifications of the NECTAr0 Chip." Internal Document Irfu CEA Saclay. v.4 2016-01-05.

[6] "Code connected - zeromq." https://zeromq.org/. Accessed: 2016-05-28.

[7] Schwanke, U. et al., "A versatile digital camera trigger for telescopes in the Cherenkov Telescope Array," NIM A 782, 92-103 (2015).

[8] "Yocto Project | Open Source embedded Linux build system, package metadata and SDK generator." https://www. yoctoproject.org/. Accessed: 2016-05-28.

[9] "Apache thrift - home." https://thrift.apache.org/. Accessed: 2016-05-28.

[10] "Protocol buffers." https://developers.google.com/protocol-buffers. Accessed: 2016-05-28.

[11] Balzer, A. et al., "The H.E.S.S. central data acquisition system," Astropart. Phys. 54, 67 (2014). 